PROCEEDINGS OF THE

AMERICAN MATHEMATICAL SOCIETY

Volume 125, Number 9, September 1997, Pages 2531-2535

S $0002-9939(97) 03894-X$

\title{
ON SOLUTIONS OF REAL ANALYTIC EQUATIONS
}

\author{
TEJINDER S. NEELON
}

(Communicated by Eric Bedford)

\begin{abstract}
Analyticity of $\mathcal{C}^{\infty}$ solutions $y_{i}=f_{i}(x), 1 \leq i \leq m$, of systems of real analytic equations $p_{j}(x, y)=0,1 \leq j \leq l$, is studied. Sufficient conditions for $\mathcal{C}^{\infty}$ and power series solutions to be real analytic are given in terms of iterative Jacobian ideals of the analytic ideal generated by $p_{1}, p_{2}, \ldots, p_{l}$. In a special case when the $p_{i}$ 's are independent of $x$, we prove that if a $\mathcal{C}^{\infty}$ solution $h$ satisfies the condition $\operatorname{det}\left(\frac{\partial p_{i}}{p y_{j}}\right)(h(x)) \not \equiv 0$, then $h$ is necessarily real analytic.
\end{abstract}

It is well known that a $\mathcal{C}^{\infty}$ function or a formal power series that satisfies a nonzero real analytic equation is necessarily real analytic. (See Corollary 2.) There are several different proofs of this fact in the literature. For example, $[6,7,10]$ contain proofs using Functional Analysis methods. Another proof follows from a theorem of Malgrange [8]. There is also an unpublished proof by Lojasiewicz [10] based on the theory of semianalytic sets.

The focus of this note is to find generalizations of this result to the case of systems of real analytic equations. More precisely, given a system of real analytic equations $p_{j}(x, y)=0,1 \leq j \leq l, x=\left(x_{1}, x_{2}, \ldots, x_{n}\right), y=\left(y_{1}, y_{2}, \ldots, y_{m}\right)$, under what conditions can one conclude that a $\mathcal{C}^{\infty}$ or formal solution $y=f(x)$ is necessarily real analytic? Such questions arise naturally in the study of reflection principles in several complex variables, see e.g. $[2,3,4,9]$. The earliest results on analyticity of formal and $\mathcal{C}^{\infty}$ solutions of real analytic equations were obtained by Artin [1] and Malgrange [8] respectively.

Since the question of analyticity is local, we will use the language of germs. Unless specified otherwise, functions, maps, spaces etc. are to be understood as germs at 0 of functions, maps, spaces etc.

Let $\mathcal{I}$ be a nonzero ideal in the ring (of germs at 0 ) of real analytic real valued functions in the variables $(x, y), x=\left(x_{1}, x_{2}, \ldots, x_{n}\right), y=\left(y_{1}, y_{2}, \ldots, y_{m}\right)$. Define $\mathrm{Jac}_{m}(\mathcal{I})$ to be the analytic ideal generated by $\mathcal{I}$ and all the $m \times m$ minors of the Jacobians, $\operatorname{Jac}\left(Q_{1}, Q_{2}, \ldots, Q_{m}\right)$, where $Q_{1}, Q_{2}, \ldots, Q_{m} \in \mathcal{I}$. Set $\mathcal{I}_{0}=\mathcal{I}$, $\mathcal{I}_{k+1}=\operatorname{Jac}_{m}\left(\mathcal{I}_{k}\right)$ for all $k \geq 0$, and $\mathcal{I}_{\infty}=\cup_{k=0}^{\infty} \mathcal{I}_{k}$. A $\mathcal{C}^{\infty}$ (resp. formal power series) map $y=f(x), f(0)=0$, is called a $\mathcal{C}^{\infty}$ (resp. formal) solution of the ideal $\mathcal{I}$ if

$$
Q(x, f(x)) \equiv 0, \forall Q \in \mathcal{I}
$$

Received by the editors August 15, 1994 and, in revised form, February 2, 1995, October 9, 1995, and March 18, 1996.

1991 Mathematics Subject Classification. Primary 14B12; Secondary 32B99.

Key words and phrases. Power series rings, real analytic equations, semianalytic sets. 
For a $\mathcal{C}^{\infty}$ map $y=f(x)$, let $\mathcal{J}_{f}$ denote the (proper) ideal in the ring of formal power series in $(x, y)$ generated by $y_{j}-F_{j}(x), 1 \leq j \leq m$, where $F_{j}$ denotes the Taylor expansion at 0 of $f_{j}$.

Theorem 1. Any $\mathcal{C}^{\infty}$ or a formal solution $y=f(x)$ of a nonzero ideal $\mathcal{I} \subset \mathcal{O}$ satisfying $\mathcal{I}_{\infty} \nsubseteq \mathcal{J}_{f}$ is necessarily real analytic.

Proof. Let $y=f(x)$ be a solution of $\mathcal{I}$, and let $k$ be an integer such that $\mathcal{I}_{k} \subseteq \mathcal{J}_{f}$ but $\mathcal{I}_{k+1} \not \subset \mathcal{J}_{f}$. By replacing $\mathcal{I}$ by $\mathcal{I}_{k}$, if necessary, we may assume that $\operatorname{Jac}_{m}(\mathcal{I}) \nsubseteq \mathbb{\mathcal { J } _ { f }}$. That means there exist $Q_{1}, Q_{2}, \ldots, Q_{m}$ in $\mathcal{I}$ such that

$$
\operatorname{det} \frac{\partial Q}{\partial\left(x^{\prime}, y^{\prime}\right)}(x, f(x)) \not \equiv 0,
$$

where $x^{\prime}$ and $y^{\prime}$ are, respectively, blocks of $p$ of the $x$-variables and $m-p$ of the $y$ variables. By reordering the $y$-variables we may assume $y^{\prime}=\left(y_{p+1}, y_{p+2}, \ldots, y_{m}\right)$. Similarly, we may assume that $x^{\prime}=\left(x_{1}, x_{2}, \ldots, x_{p}\right)$. Since $Q_{i}(x, f(x)) \equiv 0$, we have

$$
\frac{\partial Q_{i}}{\partial x_{j}}(x, f(x))+\sum_{k=1}^{m} \frac{\partial Q_{i}}{\partial y_{k}}(x, f(x)) \cdot \frac{\partial f_{k}}{\partial x_{j}} \equiv 0, \forall i, j, 1 \leq i \leq m, 1 \leq j \leq p .
$$

Define an $m \times m$ matrix

$$
T=\left[\begin{array}{cc}
\frac{\partial f}{\partial x^{\prime}} & A
\end{array}\right], A=\left[\begin{array}{c}
O_{p \times m-p} \\
-I_{m-p \times m-p}
\end{array}\right],
$$

where $O$ and $I$ denote the zero and identity matrix, respectively.

By (3), we have the following matrix equality:

$$
\frac{\partial Q}{\partial\left(x^{\prime}, y^{\prime}\right)}(x, f(x))+\frac{\partial Q}{\partial y}(x, f(x)) \cdot T \equiv 0 .
$$

Hence, by (2), we have

$$
\operatorname{det} \frac{\partial Q}{\partial y}(x, f(x)) \not \equiv 0
$$

Now we can write

$$
Q(x, y)-Q(x, z)=U(x, y, z) \cdot(y-z),
$$

where $U(x, y, z)$ is an $m \times m$ matrix with analytic entries satisfying $U(x, y, y)=$ $\frac{\partial Q}{\partial y}(x, y)$. (For example take $U(x, y, z)=\int_{0}^{1} \frac{\partial Q}{\partial y}(x, t y+(1-t) z) d t$.) By $(4)$, we get $\operatorname{det} U(x, F(x), F(x)) \not \equiv 0$. This implies that there exists an integer $k$ such that for any power series $h(x)$, we have

$$
F(x)-h(x) \equiv 0 \bmod (x)^{k} \Rightarrow \operatorname{det} U(x, F(x), h(x)) \not \equiv 0 .
$$

(Here $(x)$ denotes the ideal generated by $x_{1}, x_{2}, \ldots, x_{n}$.) Since $y=F(x)$ is a formal solution of $Q(x, y)=0$, the Artin Approximation Theorem [1, 11], when applied to the above integer $k$, yields an analytic solution $y=h(x)$ of $Q(x, y)=0$ such that $F(x)-h(x) \equiv 0 \bmod (x)^{k}$. Let

$$
d(x):=\operatorname{det} U(x, f(x), h(x)),
$$

and let $D(x)$ denote its Taylor expansion at 0 . Then $D(x) \not \equiv 0$, and hence $d(x)$ has nonzero Taylor expansion at all points near 0 . By $(5), 0=U(x, f(x), h(x)) \cdot(f-h)$, and hence $0=d(x) \cdot(f(x)-h(x))$. But the Taylor expansions of $d(x)$ at points near 
0 are all nonzero and the formal powers series expansions ring has no zero divisors. Therefore $f(x)=h(x)$ (in the sense of germs at 0), as required.

Corollary 1. If $1 \in \mathcal{I}_{\infty}$, then any $\mathcal{C}^{\infty}$ or formal solution of $\mathcal{I}$ is real analytic.

Proof. The ideal $\mathcal{J}_{f}$ is proper for any solution $f$ since, by definition of a solution, $f(0)=0$. In particular, $1 \notin \mathcal{J}_{f}$ and the corollary follows.

The condition in Corollary 1 is independent of the particular solution. In that sense Corollary 1 is a correct generalization of the result concerning a single equation.

Corollary 2 (cf. $[8,6,7,10])$. Let $f(x), x=\left(x_{1}, x_{2}, \ldots x_{n}\right), f(0)=0$, be a $\mathcal{C}^{\infty}$ function or a formal power series. If there is a nonzero real analytic function $\Phi(x, t)$ such that $\Phi(x, f(x)) \equiv 0$ then $f \in \mathcal{C}^{\omega}$.

Proof. If $\mathcal{I}$ denotes the ideal generated by $\Phi$, then $\mathcal{I}_{\infty}$ is generated by all the derivatives of $\Phi$. Since $\Phi$ is nonzero and real analytic, some derivative of $\Phi$ is nonzero at $(x, t)=(0,0)$. Hence $1 \in \mathcal{I}_{\infty}$, and Corollary 1 applies.

The condition in Theorem 2 below, although solution specific, is natural and is easy to verify for a given solution.

Theorem 2. Let $h: \mathbb{R}^{n} \longrightarrow \mathbb{R}^{m}, h(0)=0$, be a $\mathcal{C}^{\infty}$ map, and let $q: \mathbb{R}^{m} \longrightarrow \mathbb{R}^{m}$, $q(0)=0$, be a real analytic map. Let $\triangle=\operatorname{det}\left(\frac{\partial q_{j}}{\partial \xi_{k}}\right)_{m \times m}$. If the following two conditions are satisfied

(i) $q \circ h \in \mathcal{C}^{\omega}\left(\mathbf{R}^{n}\right)$,

(ii) $\triangle \circ h \not \equiv 0$, then $h \in \mathcal{C}^{\omega}\left(\mathbf{R}^{n}\right)$.

Proof. Observe that if $\Delta(h(p)) \neq 0$ for some $p$, then by applying the Implicit Function Theorem to $q(\xi)=q(h(x))$, we conclude that $\xi=h(x)$ is the unique real analytic solution near $p$. Hence $h$ is real analytic in the set $\{x: \Delta(h(x)) \neq 0\}$.

Case I. $h \sim 0$. (The symbol $\sim$ means that the Taylor expansion at the origin is zero.)

We will show that $h \equiv 0$ in this case.

By (i), we have $q(h(x)) \equiv 0$. If $p_{0}$ is any fixed point such that $\triangle\left(h\left(p_{0}\right)\right) \neq 0$, then, by arguing as in the above observation, we conclude that $h(x)=h\left(p_{0}\right)$ for all $x$ near $p_{0}$.

Hence, the set

$$
U=\left\{x: h(y)=h\left(p_{0}\right) \forall y \text { near } x\right\}
$$

is nonempty and is clearly open. We claim that $U$ is also closed. Indeed, by the continuity of $h$, we have for any $\tilde{x}$ in the closure of $U, h(\tilde{x})=h\left(p_{0}\right)$. Also, we have $\triangle(h(\tilde{x}))=\triangle\left(h\left(p_{0}\right)\right) \neq 0$, and hence $\tilde{x} \in U$. Hence $U=\mathbb{R}^{n}$. Since $h(0)=0$, we have $h(x)=0$ for all $x$.

Case II. $h \nsim 0$.

We claim that it is enough to prove that $\triangle \circ h \not 0$. Indeed, if $\triangle(H(x)) \not \equiv 0$, where $H$ is the Taylor expansion of $h$ at 0 , then Theorem 1 implies that $h(x)$ is real analytic.

By an appropriate rotation around 0 , we may assume that $\delta(t)=\triangle \circ h_{0}(t) \not \equiv 0$ where $h_{0}(t)=h(t, 0, \ldots, 0)$. It is enough to prove that $\delta \not 0$. Since $\delta(t) \not \equiv 0$ near 
0 , by making the transformation $t \rightarrow-t$, if necessary, we may assume that $\delta(t) \not \equiv 0$ on $[0, \varepsilon]$ for all $\varepsilon>0$.

Lemma. There exists an $\varepsilon>0$ such that $\delta(t) \neq 0$ for all $t \in(0, \varepsilon]$.

Proof. Since the function $q \circ h$ is real analytic, the set

$$
Z=\left\{(\xi, t) \in \mathbb{R}^{m} \times(0, \varepsilon): q(\xi)=q \circ h(t)\right\}
$$

is semianalytic. Let $Z^{\prime}$ denote the set all smooth points $\zeta \in Z$ with $\operatorname{dim}_{\zeta}(Z)=1$. (All results about semianalytic sets used here can be found in [5].)

Let $J \subset(0, \varepsilon)$ be any open interval such that $\delta(t) \neq 0$ for all $t \in J$. Observe that the Jacobian determinant of the map

$$
\mathbb{R}^{m} \times J \ni(\xi, t) \rightarrow(q(\xi), t)
$$

at a point $\left(h_{0}(t), t\right)$ is equal to $\delta(t)$. Hence, the above map is a real analytic diffeomorphism of a neighbourhood of the graph of $h$ over $J$ onto a neighbourhood of the graph of $q \circ h$ over $J$. We conclude that $\left(h_{0}(t), t\right) \in Z^{\prime}$ for all $t$ where $\delta(t) \neq 0$.

Note that $\delta(t)$ real analytic on the set $\{t: \delta(t) \neq 0\}$ because $h_{0}(t)$ is real analytic there. Since $Z^{\prime}$ is a 1 -dimensional smooth semianalytic set, $Z^{\prime \prime}=Z^{\prime} \cap\{(\xi, t)$ : $\Delta(\xi) \neq 0\}$ is also a semianalytic set. Observe that a connected component of the graph of $h$ over the set $\{t \in(0, \varepsilon): \delta(t) \neq 0\}$ is also a connected component of $Z^{\prime \prime}$. Since the family of connected components of a semianalytic set is locally finite, $\delta(t)$ can have only a finite number of zeroes in $(0, \varepsilon)$. By choosing $\varepsilon>0$ sufficiently small, the lemma follows.

Let $\varepsilon>0$ such that $\delta(t) \neq 0$ for all $t \in(0, \varepsilon]$.

The graph of $h$ over $(0, \varepsilon]$, being a connected component of $Z^{\prime \prime}$, is a semianalytic set. Hence, the closure

$$
G=\left\{(\xi, t) \in \mathbb{R}^{m} \times[0, \varepsilon]: \xi=h_{0}(t)\right\},
$$

is also semianalytic.

We claim that it is enough to restrict to the case when $h$ satisfies

$$
\left|h_{0}(t)\right| \geq|t| \forall t \text { near } 0 .
$$

Indeed, if we put $h^{\star}(x)=(h(x), x)$ and $q^{\star}(\zeta)=(q(\xi), \tau), \zeta=(\xi, \tau), \tau \in \mathbb{R}$, then $q^{\star} \circ h^{\star} \in \mathcal{C}^{\omega}$. Clearly $\left|h^{\star}(t)\right| \geq|t|$ near 0 , where $h_{0}^{\star}(t)=\left(h_{0}(t), t\right)$, and moreover, $\delta(t)=\operatorname{det}\left(\frac{\partial q^{\star}}{\partial \zeta}\right)\left(h_{0}^{\star}(t)\right)$ for all $t$.

By the lemma, the intersection of the one-dimensional semianalytic set $G$ with the analytic set $V=\{\xi: \Delta(\xi)=0\}$ is $0=h_{0}(0)$. By a basic inequality (due to Lojasiewicz) in the theory of semianalytic sets, there are a constant $C_{1}>0$ and an integer $l_{1}>0$, see e.g. [5], such that

$$
\operatorname{dist}\left(h_{0}(t), V\right) \geq C_{1}\left(\operatorname{dist}\left(h_{0}(t), G \cap V\right)\right)^{l_{1}}=C_{1}\left(\operatorname{dist}\left(h_{0}(t), 0\right)\right)^{l_{1}} .
$$

Also by Lojasiewicz's Inequality, there are a constant $C_{2}>0$ and an integer $l_{2}>0$ such that

$$
|\Delta(\xi)| \geq C_{2} \cdot \operatorname{dist}(\xi, V)^{l_{2}} .
$$

By combining (6), (7), and (8), we conclude that there are a $t_{0}>0, C>0$, and an integer $l>0$ such that

$$
|\delta(t)| \geq C|t|^{l}, \quad \forall t \in\left[0, t_{0}\right] .
$$


Since $\delta$ is $\mathcal{C}^{\infty}$, it is easy to see that above inequality implies that the $k$-th derivative $\delta^{(k)}(0) \neq 0$ for some $k, 0 \leq k \leq l$.

Remark 1. Above results are no longer true if the hypothesis $f \in \mathcal{C}^{\infty}\left(\mathbf{R}^{n}\right)$ is replaced by $f \in \mathcal{C}^{l}\left(\mathbf{R}^{n}\right), l<\infty$. For example, take $\phi(y)=y^{2}$ and $f(x)=x^{\frac{5}{2}}$.

Remark 2. [5] contains the following interesting and related result:

Let $h=\left(h_{1}, h_{2}, \ldots, h_{m}\right)$, where $h_{j}(x), 1 \leq j \leq n$, are convergent power series in $n$ variables, and $Q(y)$ a formal power series in $m$ variables. If the generic rank of $h$ is $m$, then

$$
Q(h) \text { convergent } \Rightarrow Q \text { convergent . }
$$

\section{ACKNOWLEDGMENT}

I wish to thank the referee for the proof of the Case II in Theorem 2, and many other helpful suggestions.

\section{REFERENCES}

1. Artin, M., On the Solutions of Analytic Equations. Invent. Math. 5 277-291 (1968). MR 38:344

2. Baouendi, M. S., and Rothschild, L. P., Images of real hypersurfaces under holomorphic mappings. J. Differential Geometry 36 (1992), 75-88. MR 94i:32028

3. Baouendi, M. S., H. Jacobowitz and F. Treves, On analyticity of CR mappings. Ann. Math. 122 (1985), 365-400. MR 87f:32044

4. Baouendi, M. S. and L. P. Rothschild, Germs of CR maps between real analytic hypersurfaces Invent. Math. 93 (1988) no. 3, 481-500. MR 90a:32036

5. Bierstone, E. and P. D. Milman, Semianalytic and Subanalytic sets IHES Publ. Math.67 Paris 1988, 5-42. MR 89k:32011

6. Bochnak, J. Analytic Functions in Banach Spaces. Studia Mathematica, T. XXXV.(1970).

7. Bochnak, J. and J. Siciak Analytic Functions in Topological Vector Spaces. Studia Mathematica, T. XXXIX.(1971). MR 47:2365

8. Malgrange, B., Ideals of differentiable functions. Tata Institute of Fundamental Research, Bombay, Oxford University Press, 1966. MR 35:3446

9. Neelon, T. S.Holomorphic Extensions of CR Functions and CR Mappings Ph. D. thesis, Rutgers, The State University of New Jersey, New Brunswick, NJ 08901. (1993)

10. Siciak, J. A Characterization of Analytic Functions of $n$ Real Variables. Studia Mathematica, T. XXXV.(1970). MR 43:4986

11. Tougeron, J. C., Ideaux de fonctions differnetiables, Springer-Verlag, 1972.

College of Arts and Sciences, California State University San Marcos, San Marcos, CALIFORnia 92096

E-mail address: neelon@mailhost1.csusm.edu 